\title{
La mise en œuvre d'un plan de prévention des risques technologiques: acceptabilité économique vs réduction des risques
}

\author{
Marie-Gabrielle Suraud* \\ Sciences de la communication, UMR5044 CERTOP, CNRS, UT2J, UPS, Toulouse, France
}

Reçu le 14 août 2017. Accepté le 7 novembre 2018

Depuis la catastrophe d'AZF, survenue à Toulouse en 2001, l'arsenal législatif en matière de prévention des risques technologiques et naturels majeurs s'est étoffé. L'article analyse les négociations entourant les plans de prévention des risques des usines classées «Seveso seuil haut», instaurés par la loi Bachelot de juillet 2003. Si une négociation a lieu au sujet de l'estimation des dangers qui est réalisée par les entreprises, ce n'est pas le cas quand ces dernières convoquent le principe de ce qui est «économiquement acceptable » de faire en matière de prévention des risques; un principe qui est aussi invoqué par l'administration pour éviter de faire déménager des entreprises situées dans des zones industrielles portuaires à risque. Celui-ci apparaît donc, selon l'auteur, comme «une sorte de voile dont il n’est souhaité par personne qu'il soit levé».

La Rédaction

Résumé - Ce texte s'intéresse à la loi Bachelot (juillet 2003) et à la mise en œuvre d'un plan de prévention des risques technologiques majeurs (PPRT) dans une zone industrialo-portuaire. D'une part, on peut observer que la notion d'acceptabilité économique, qui permet aux entreprises de faire face aux exigences administratives, est réglementaire mais pas réglementée. En conséquence, la loi destinée à protéger l'industrie et les zones urbaines engendre finalement une tension entre activités économiques Seveso et non Seveso. D'autre part, ce texte montre comment, pour sortir de ce blocage, les décideurs locaux s'engagent dans une démarche d'adaptation de la loi Bachelot que l'État accepte de déformer, à travers des arrangements avec des décideurs locaux.

Mots-clés : risques industriels / acceptabilité économique / réglementation / PPRT / zone industrialoportuaire

\begin{abstract}
The implementation of a technological risks prevention plan: economic acceptability vs. risk reduction. The problem of industrial disaster risks was raised at the earliest stages of industrialization and has been addressed, particularly since the 1970s, by European regulations, i.e. the Seveso directives and their national adaptation. Following the disaster at the AZF plant in Toulouse (September 2001), a specific text was adopted by the French State: The Major Technological and Natural Risks Act, commonly known as the "Bachelot Act" (July 2003). This act aims, notably, to implement Technological Risks Prevention Plans (PPRTs) in "upper-tier Seveso" sites. In this perspective, we deal with the shaping of a PPRT in an industrial port area, that of Le HavreGonfreville. The analysis of the conditions, forms and effects of the setting-up of the PPRT leads to two conclusions. On the one hand, we observe that the notion of "economic acceptability", which allows companies to challenge administrative requirements, although regulatory, is not defined by any specific regulation. As a result, the Bachelot Act designed to protect urban areas, ultimately creates a tension
\end{abstract}

\footnotetext{
*Auteur correspondant : marie-gabrielle.suraud@iut-tlse3.fr
} 
between Seveso and non-Seveso economic activities. On the other hand, our paper shows that, in order to defuse this tension, local decision-makers engaged in a process of distorting the Bachelot Act, which was accepted by the State through local arrangements. In the end, in such an industrial port area, the Bachelot Act had the effect of tightening risk prevention, but not without coming up against the limits set by the undefined notion of economic acceptability.

Keywords: industrial risks / economic acceptability / risks regulation / PPRT / industrial port area

Ainsi que l'ont montré de nombreuses recherches, la protection de l'industrie a été, pour l'État central français, un enjeu constant de la réglementation des risques industriels (Chabbal, 2005). Le fameux décret napoléonien de 1810 , à l'origine de la création des «établissements classés» (dangereux, insalubres et incommodes), permet à l'État d'encadrer les conditions d'implantation des fabriques. Il limite, sans la contrôler complètement, la portée des contestations locales d'industriels ou de propriétaires fonciers refusant la dévalorisation de leurs activités ou de leurs biens du fait des nuisances, des pollutions ou des menaces d'accidents (Fressoz, 2012; Lascoumes, 2011; Le Roux et Letté, 2013 ; Rasse, 2009). Il trace le chemin d'un peu-disant réglementaire retirant le plus d'entraves possible à l'expansion de l'industrie.

Succédant à ce protectionnisme intérieur aux conséquences écologiques négatives, les réglementations européennes et nationales du dernier tiers $\mathrm{du} \mathrm{xx}^{\mathrm{e}}$ siècle infléchissent lentement la tendance précédente. Elles répondent à la montée des contestations de la pollution environnementale et des risques d'accident, contestations alimentées par la répétition de catastrophes (Centemeri, 2010). Ces réglementations traduisent un accroissement graduel des exigences de prévention du risque technologique majeur à travers les directives Seveso (en 1982 et ultérieurement). Elles tentent ainsi de contenir une urbanisation qui rattrape les usines (Bonnaud, 2005; Bonnaud et Martinais, 2005) et de limiter la "gravité» des événements catastrophiques potentiels. Ces réglementations marquent des avancées, mais souvent limitées ou partielles. Par exemple, en l'absence d'une réglementation adéquate sur les transports de matières dangereuses, les établissements industriels contournent les seuils de stockage imposés par les directives, en jouant sur le volume et la fréquence des approvisionnements. Des gares de triage deviennent ainsi de très gros centres de stockage provisoire de matières dangereuses, sans que pour autant une réglementation Seveso ne s'y applique au même titre qu'aux usines.

Une troisième période émerge après et à cause de la crise sociale née de la catastrophe de l'usine AZF de Toulouse (septembre 2001). L'État fait voter une loi, généralement appelée loi Bachelot (juillet 2003), relative à la prévention des risques technologiques (et naturels) majeurs et à la réparation des dommages. Elle se concrétise dans des plans de prévention des risques technologiques (PPRT) autour des usines dites « Seveso seuil haut» (Martinais, 2007).

Cette nouvelle réglementation est fondée sur l'idée de limiter l'urbanisation autour des usines à risques, donc sur la nécessité de transformer leur espace environnant. Une telle idée engendre logiquement un double mouvement: «faire le vide» autour des établissements Seveso seuil haut, les territoires riverains étant alors soumis à des mesures d'expropriation, de délaissement ou de préemption (Gralepois, 2011); imposer à ces entreprises des transformations visant la réduction des risques à la source afin de limiter la surface des territoires ainsi vidés et gelés (Frère et Gibout, 2012).

La réglementation Bachelot protège cependant les entreprises à risques en mettant, de fait, à leur disposition la notion "d'économiquement acceptable», une opportunité qui leur permet de poser des bornes aux mesures de réduction des risques à la source qui leur sont demandées par l'administration. Les entreprises peuvent ainsi opposer aux injonctions de la police des installations un argument d'inacceptabilité économique du point de vue de leur survie.

Cette notion joue un rôle incontestable dans les négociations entre contrôleurs et contrôlés, sans que ce rôle n'ait été explicité dans la littérature scientifique. L'inacceptabilité économique a certes été envisagée à travers sa calculabilité (Propeck-Zimmermann et al., 2007) ou sa portée éventuelle pour la décision à travers des analyses coûts-bénéfices (Treich, 2008); cependant, le processus sociologique dont elle est l'expression n'a guère fait l'objet de recherches.

L'objectif de ce texte ${ }^{1}$ est d'examiner ce processus sociologique, c'est-à-dire la dynamique des contestations, négociations et décisions passant par la référence à l'acceptabilité économique. En ce sens, cette notion est prise ici non pas comme une grandeur économique, mais comme une source de tensions dont les modalités de

\footnotetext{
${ }^{1}$ Qui résulte d'une opération financée par la Foncsi (Fondation pour une culture de la sécurité industrielle), 2012-2015.
} 
ré-appropriation et de requalification par les différentes parties prenantes se construisent sur le terrain.

En effet, l'acceptabilité économique est réglementaire sans être réglementée: l'acceptabilité économique est réglementaire puisqu'inscrite dans les circulaires relatives à la prévention des risques, mais pas réglementée dans la mesure où les critères qui pourraient présider à son évaluation ne sont pas formellement définis par la réglementation. Dans ces conditions, l'évaluation de l'acceptabilité économique reste à la discrétion des entreprises Seveso qui ont recours à ce principe pour desserrer les contraintes de l'État. Bien qu'argumenté et justifié auprès des autorités administratives, l'argument économique n'en reste pas moins déterminé unilatéralement par les entreprises dans la mesure où l'administration n'a aucun moyen d'évaluer la justesse des données fournies par les entreprises.

Le recours à l'acceptabilité économique par les entreprises Seveso a pour effet de limiter les efforts en termes de réduction des risques à la source. Afin de préserver l'activité des entreprises non Seveso situées en zone de risques à «vider», les décideurs locaux ${ }^{2}$ ont, à leur tour, recours au principe d'économiquement acceptable. Afin de débloquer une situation dans laquelle est mis en regard l'intérêt économique des entreprises Seveso et l'intérêt économique des entreprises non Seveso, les décideurs locaux s'associent pour inciter l'État à déformer la réglementation Bachelot.

Cet article s'appuie sur un travail de recherche mené entre 2012 et 2016 sur la zone industrialo-portuaire (ZIP) du Havre-Gonfreville (Seine-Maritime), dont le PPRT représente un cas d'école en France. En effet, ce site:

- regroupe un nombre élevé d'établissements «Seveso seuil haut» de pétrochimie et chimie (16 établissements), faisant de ce PPRT le plus conséquent de France;

- concentre de nombreuses activités économiques non Seveso (plus de 250 entreprises de toutes tailles sont répertoriées), riveraines des entreprises Seveso et ainsi directement concernées par le PPRT;

- engage le territoire du Grand port maritime du Havre (GPMH), ainsi que les activités économiques qui y sont installées ;

- concerne un tissu urbain dense intégrant six communes, dont la ville du Havre elle-même.

Dans l'ensemble de la recherche, plus d'une soixantaine d'entretiens ont été menés de 2011 à 2016, auprès d'élus, d'industriels, de fonctionnaires de services déconcentrés de l'État, de membres d'associations pour

\footnotetext{
${ }^{2}$ Mairie du Havre, Codah (Communauté de l'agglomération havraise), $\mathrm{CCIH}$ (Chambre de commerce et d'industrie du Havre) et GPMH (Grand port maritime du Havre).
}

l'environnement, de représentants des organisations syndicales d'entreprises Seveso, de membres d'associations de riverains, de responsables du GPMH, d'agents des collectivités territoriales. À ces entretiens, dont une partie est directement exploitée pour rédiger ce texte, s'ajoute l'assistance à de nombreuses réunions, institutionnelles ou associatives.

Au Havre, bien que les enjeux liés à l'habitat aient suscité des réactions de la part des élus et des associations, l'impact des mesures PPRT sur l'activité économique non Seveso a progressivement représenté un enjeu majeur. Bien que d'autres territoires présentent des caractéristiques géographiques (présence d'un port) et industrielles (plusieurs unités Seveso regroupées et un tissu industriel riverain important) à première vue comparables, Le Havre a représenté un terrain d'étude spécifique à plusieurs égards. D'une part, c'est le PPRT du Havre qui regroupe le plus grand nombre d'entreprises Seveso (seize) impactant le tissu industriel riverain non Seveso le plus important. D'autre part, le port du Havre tient une place importante dans l'histoire de l'économie locale et pèse encore aujourd'hui fortement sur le développement économique de la région. À ce titre, la nécessité de maintenir les activités portuaires (situées dans la zone de risques) a joué un rôle clé dans les négociations. Enfin, les négociations entre les décideurs locaux (port, CCIH, élus, Dreal ${ }^{3}$, notamment) ont été marquées au Havre par la volonté de «travailler ensemble» pour faire valoir auprès du ministère de l'Écologie les caractéristiques spécifiques du territoire.

La situation havraise étant à l'origine d'une réflexion nationale visant à «aménager» certains aspects de la loi de 2003 et à modifier les conditions de son application, elle fait de ce territoire et de la dynamique locale un terrain d'étude pertinent pour l'analyse des évolutions réglementaires post-2003. L'ampleur des évolutions localement envisagées a conduit à repousser l'approbation du PPRT du Havre pendant plusieurs années.

Nous mettrons en évidence, dans un premier temps, de quelle façon le principe «d'économiquement acceptable » pèse sur les négociations relatives au mode de calcul des risques entre l'administration et les entreprises Seveso (première phase de négociations correspondant à la construction des matrices MMR, ou mesure de maîtrise des risques). Nous montrerons, dans un second temps, comment l'appropriation par les décideurs locaux du principe d'économiquement acceptable pour conserver le tissu industriel non Seveso a conduit à une double démarche: d'une part, les entreprises Seveso consentent à de nouveaux efforts en termes de réduction des risques à la source au-delà du cadre MMR ; d'autre part, le travail

\footnotetext{
${ }^{3}$ Direction régionale de l'environnement, de l'aménagement et du logement.
} 
collaboratif des décideurs locaux incite l'État à réviser la réglementation et bouscule significativement les principes initiaux inscrits dans la loi Bachelot (seconde phase de négociation post-MMR, dépassant le cadre réglementaire).

\section{Risque technologique majeur vs risque économique: des calculs incommensurables}

Adoptée «à chaud» peu après la catastrophe de l'usine AZF, la loi Bachelot se présente comme une loi expérimentale dont les enjeux ne sont immédiatement perçus que par un petit cercle d'initiés. Un certain nombre de responsables politiques, essentiellement les élus des communes concernées, considèrent qu'elle s'éteindra d'elle-même tant sa mise en œuvre paraît hors de portée. Pourtant, après quelques années de tâtonnements, qui reflètent la difficulté de l'administration de contrôle à mettre en œuvre la nouvelle réglementation (Martinais, 2007; Martinais et Galland, 2010), une circulaire du ministère de l'Écologie, datée du 10 mai $2010^{4}$, propose un cadre méthodologique général, traduisant la stabilisation des éléments de doctrine. Cette circulaire compile les règles proposées dans différents textes réglementaires entre 2005 et 2010 et relatives: à la rédaction des études de dangers, aux critères d'appréciation de la démarche de réduction du risque à la source, engagée par les entreprises, et à la politique de maîtrise de l'urbanisation autour des sites industriels. Affichant la réduction des risques à la source comme une priorité, la circulaire définit ainsi des critères précis - et contraignants - d'évaluation et de gestion du risque technologique majeur.

La pression exercée par l'État peut donc s'accroître grâce à de nouveaux leviers incarnés par une méthodologie nouvelle et sophistiquée de prévention des risques. Cependant, si la circulaire de 2010, à visée harmonisatrice, va conduire les entreprises à assumer la prise en charge financière des réductions du risque à la source, le critère de «l'économiquement acceptable» leur fournit un moyen de contrôler le niveau d'exigence de l'État.

\section{Des désaccords sur les modes de calcul des probabilités...}

La loi Bachelot et ses décrets d'application prévoient une première phase de négociations entre administration de l'environnement et entreprises, à partir des études de dangers réalisées par ces dernières. Les critères d'appréciation des risques - ou critères MMR - sont au centre de cette première phase. La « démarche MMR », située au

\footnotetext{
$\overline{{ }^{4} B O \text { du MEEDDM n }}{ }^{\circ} 2010 / 12$ du 10 juillet 2010.
}

cœur de la méthodologie réglementaire, apparaît comme un «indicateur de compatibilité de l'entreprise avec son environnement ${ }^{5} »$.

Concrètement, cette démarche se traduit par la réalisation d'une matrice à deux entrées : «gravité» et « probabilité » d'occurrence d'un événement, impliquant qu'un phénomène dangereux dont la combinaison de la gravité et de la probabilité est trop élevée devra faire l'objet d'une réduction des risques à la source. Le positionnement de l'ensemble des phénomènes dangereux dans cette matrice, à cinq classes de probabilité et de gravité, permet à l'administration de statuer sur «l'acceptabilité ${ }^{6} »$ du niveau de maîtrise du risque par l'entreprise.

Lors de cette première phase, le paramètre " gravité », quoique toujours discutable, ne fait l'objet ni de désaccords ni même de débats entre l'administration et les entreprises. En revanche, les calculs de probabilité soulèvent des questions en raison de l'existence de nombreuses méthodologies et modèles de calcul, tant en France qu'au niveau international. Si certains groupes industriels disposent d'un savoir-faire propre et des méthodes clairement identifiables, tel n'est pas le cas pour d'autres entreprises, ce qui les oblige à aller chercher une méthode "clé en mains" chez un prestataire. Bien que soient disponibles des méthodes internationales fournies par des cabinets d'expertise, basés dans des pays où le probabilisme est bien ancré (aux Pays-Bas, par exemple), aucune méthode ne s'impose particulièrement, ce qui engendre des différences de traitement entre les entreprises : «Pour un débit de fuite, prendre 10 ou $30 \%$, ça change complètement le résultat. Certaines entreprises se pénalisaient trop par rapport à celles qui prenaient des critères plus souples ${ }^{7}$.»

Or, dans le cadre de la démarche MMR, l'enjeu de la détermination des classes de probabilité est central, parce qu'il donne la possibilité d'exclure des phénomènes dangereux dont la gravité est très élevée, mais dont la probabilité d'occurrence est estimée comme étant extrêmement faible. L'exclusion d'un phénomène dangereux évalué comme étant extrêmement peu probable peut engendrer des effets sur la construction du PPRT. La suppression du risque associé à un événement peut, le cas échéant, libérer une zone (plus ou moins importante) potentiellement soumise à expropriation ou à délaissement. Cependant, l'exclusion d'un phénomène dangereux nécessite un accord formel de l'administration, ce qui implique que les principes de calcul de probabilité adoptés par les entreprises et par l'administration soient sinon identiques, tout au moins compatibles: "Pour

\footnotetext{
$\overline{{ }^{5} \text { Circulaire du } 10 \text { mai } 2010 .}$

${ }^{6}$ Circulaire du 10 mai 2010, p. 2.

${ }^{7}$ Agent de la Dreal 1, mars 2013
} 
exclure un phénomène dangereux, il faudrait qu'il soit exclu suivant des règles qui sont préétablies et publiques, que ça ne soit pas une boîte noire. Par exemple, avant 2005 , on ne pouvait pas savoir si certaines sources de risque avaient été supprimées par l'entreprise ${ }^{8}$.»

Durant cette première phase, qui s'étend de 2005 à 2010, l'administration doit se forger une expérience de la démarche probabiliste dont elle ne disposait pas initialement puisqu'elle était surtout rompue au déterminisme de l'accident majorant. Néanmoins, l'administration refuse d'accorder un blanc-seing aux modèles proposés par les entreprises et, ce faisant, doit faire face à une double difficulté. D'une part, la Dreal ne peut avoir accès aux modèles de calcul des probabilités utilisés par les entreprises: "On n'arrivait pas à savoir, et encore moins lorsque c'était un prestataire de services extérieur qui réalisait les calculs de probabilité ${ }^{9}$.» D'autre part, l'administration conteste un des fondements essentiels de certaines méthodes de calcul : «Sur ce phénomène, on n'a pas suffisamment d'événements et de retour d'expérience pour évaluer la probabilité avec sérieux ${ }^{10}$. »

Dans le cas de la ZIP duHavre-Gonfreville, ces négociations prennent une dimension particulière du fait du grand nombre d'établissements à risques sur le site et de leur proximité avec les communes riveraines, dont celle du Havre. L'administration engage de multiples négociations serrées avec chacune des entreprises qui contestent la validité des calculs de probabilité proposés par l'État : «Sur une probabilité infinitésimale, leur tiersexpert [mandaté par l'administration] a conclu qu'on ne pouvait pas exclure le phénomène. On n'a pas réussi à valoriser nos joints indestructibles. Donc, on n'a pas pu descendre en classe de probabilité ${ }^{11}$. » Les tensions sont parfois telles que, pour échapper à des négociations confinées avec les industriels et n'aboutissant pas à une solution partagée, la Dreal a recours à la mise en public des désaccords: «Du coup, ça a été présenté en Clic [comité local d'information et de concertation] pour faire état des divergences méthodologiques [sur les calculs de probabilité] qu'on avait avec certaines entreprises ${ }^{12}$. »

Ainsi, entre 2005 et 2010, afin d'harmoniser et officialiser les méthodes de calcul de probabilité d'occurrence des phénomènes dangereux s'instaure une longue et laborieuse période de négociations et d'allers-retours entre l'État (notamment par le biais de la Direction générale de la prévention des risques: DGPR, du ministère de l'Écologie) et les principaux groupes industriels. Les tensions entre l'administration et les

\footnotetext{
${ }^{8}$ Agent de la Dreal 2, janvier 2013.

${ }^{9}$ Agent de la Dreal 2, janvier 2013.

${ }^{10}$ Agent de la Dreal 1, mars 2013.

${ }^{11}$ Entretien avec un industriel 1, avril 2014.

${ }^{12}$ Agent de la Dreal 3, mars 2015.
}

industriels amènent l'État à compléter la réglementation et à produire, en 2010, une circulaire visant à l'harmonisation des modèles d'évaluation des risques.

L'harmonisation des méthodes de calcul des probabilités permet à la Dreal de sortir de situations de blocage, puisque certaines entreprises, dont les risques impactaient une surface urbaine importante, refusaient de procéder à des réductions de risque à la source en rejetant les calculs de probabilité avancés par l'administration. Cette démarche a ainsi fourni à l'administration la capacité de remettre en cause et de peser sur les évaluations de risques réalisées par chacune des entreprises Seveso: «Ce qu'a permis AZF, c'est de nous doter de moyens pour vérifier les barrières de sécurité mises en place par l'industriel. C'est la grande avancée de cette loi : introduire le concept de réduction des risques à la source et nous permettre de peser sur l'industriel... et de façon bien plus importante que ce que nous avions imaginé au début de l'exercice PPRT ${ }^{13}$.»

Progressivement, ces démarches ont conduit à des réductions significatives de l'espace impacté autour des entreprises Seveso. Par exemple, un des rayons engendrés par une entreprise est ramené de $7 \mathrm{~km}$ à $2,2 \mathrm{~km}$, réduisant fortement le nombre d'activités et d'habitats susceptibles d'être supprimés (en particulier, une grande partie de l'agglomération du Havre). Pour autant, ainsi qu'on le verra en seconde partie de ce texte, la surface restant impactée regroupe plusieurs centaines d'entreprises non Seveso, révélant les lacunes du cadre réglementaire fondé sur la construction des MMR.

En effet, malgré les avancées, dans la région havraise, la surface affectée reste encore très importante et représente un problème majeur pour des territoires à forte activité économique sommés de se conformer aux mesures d'expropriation et de délaissement. Ainsi, malgré la production d'une méthodologie d'évaluation des risques harmonisée, ce processus s'est accompagné du recours au principe d'acceptabilité économique. Ce principe a fourni, dans la pratique, un argument de poids aux entreprises Seveso et leur a accordé des marges de manœuvre pour «ajuster» les efforts à consentir réglementairement en termes de réduction de risques à la source.

On montrera dans ce qui suit comment et pourquoi le principe d'économiquement acceptable a pesé de façon aussi significative que la construction des MMR.

\section{... au principe «d'économiquement acceptable »}

Le recours au principe d'acceptabilité économique est très antérieur à la loi Bachelot: «[l'étude de dangers] justifie que le projet permet d'atteindre, dans des

${ }^{13}$ Agent de la Dreal 1, mars 2013. 
conditions économiquement acceptables, un niveau de risque aussi bas que possible, compte tenu de l'état des connaissances et des pratiques et de la vulnérabilité de l'environnement de l'installation ${ }^{14}$.» $\mathrm{Au}$ cours des années 1990 , ce principe est couramment invoqué par les entreprises pour justifier des refus opposés aux suggestions, incitations ou demandes, exprimées par la police des installations classées pour réduire le niveau des risques. Si de nouvelles règles de calcul des risques sont désormais opposables par l'administration aux entreprises, des énoncés de la circulaire de 2010 maintiennent cependant l'orientation antérieure: «Le projet [de réduction des risques] permet d'atteindre, dans des conditions économiquement acceptables, un niveau de risque aussi bas que possible, compte tenu de l'état des connaissances et des pratiques et de la vulnérabilité de l'environnement de l'installation ${ }^{15}$.» La circulaire réaffirme ainsi la nécessité de limiter les mesures de maîtrise des risques si l'entreprise considère sa survie compromise.

Si ce principe tient une place importante dans les négociations, il n'est jamais explicité dans une formulation qui serait aussi précise que les calculs de probabilité et qui pourrait servir de base aux négociations entre la Dreal et les entreprises. En pratique, ce principe, non quantifié, donc difficilement négociable, permet de façon unilatérale à une entreprise de mettre un plafond au niveau d'effort qu'elle pourrait consentir en termes de réduction des risques à la source. Paradoxalement, alors même que cette notion pèse réglementairement dans la démarche d'évaluation des risques, elle relève entièrement de l'appréciation singulière des entreprises. L'acceptabilité économique est une sorte de voile dont il n'est souhaité par personne qu'il soit levé.

Par conséquent, cette situation pose la question du contrôle du caractère économiquement inacceptable des mesures de sécurisation demandées aux entreprises, une question accentuée par la démarche probabiliste de maîtrise des risques : « Si on regarde bien la circulaire de 2010 , on nous demande de réduire les risques à la source à la limite de ce qui est économiquement acceptable, c'est-à-dire indépendamment du critère $\mathrm{MMR}^{16}$ !»

Coexistent ainsi deux types de critères incommensurables entre eux. Alors que l'équation du risque «probabilité $\times$ gravité » peut être un objet de négociation entre contrôlé et contrôleur, conduisant les deux parties à des échanges techniques, l'acceptabilité économique, quant à elle, échappe à cette négociation parce que l'administration ne dispose pas des compétences pour en discuter: «Bien évidemment, là, on a des difficultés pour

\footnotetext{
${ }^{14}$ Extrait de l'article 3 du décret du 21 septembre 1977.

${ }^{15}$ Article R. 512-9 du Code de l'environnement, guide p. 132.

${ }^{16}$ Agent de la Dreal 3, mars 2015.
}

estimer ce qui est économiquement possible. Ce n'est pas notre métier, et c'est de toute façon très complexe. On n'est pas capable de le dire ${ }^{17}$. $\gg$ L'administration est de ce point de vue démunie et n'est plus en mesure de négocier, au sens propre, sur cette base : «... donc on retourne voir les industriels et on leur dit: "voilà, est-ce que vous n'avez pas encore des possibilités pour réduire au-delà de ce que vous avez jugé économiquement acceptable ? ${ }^{18}$ "

Il apparaît donc une asymétrie entre, d'une part, la sophistication des règles méthodologiques organisant le calcul des risques et, d'autre part, l'absence de référentiel pour contrôler le calcul économique de la réduction des risques par les entreprises. Cette asymétrie conduit à interroger le caractère acceptable de "l'acceptabilité économique », surtout dans un contexte où l'application de la loi Bachelot, visant non plus seulement à pétrifier des territoires mais à faire le vide autour des usines à risques, représente un véritable enjeu pour l'existence et le développement des territoires.

L'étendue des zones restant affectées malgré la circulaire de 2010 n'a donc pas représenté un argument suffisant pour obtenir une réduction supplémentaire des risques à la source de la part des entreprises : «Il y a la théorie et la pratique. Dans de nombreux cas - et j'en ai plusieurs en tête - on fait une MMR acceptable et après, hop !, nous voilà partis à faire le PPRT. Mais quand on fait le PPRT, on s'aperçoit qu'il y a des choses inacceptables socialement... et on trouve d'autres solutions qui vont au-delà de ce que la MMR a validé antérieurement. Plusieurs fois, ça s'est produit ${ }^{19} \ldots$... Les entreprises se réfèrent à l'inacceptabilité économique des mesures adéquates et renvoient aux autorités locales la prise en charge financière de mesures de protection s'avérant nécessaires: " $\mathrm{Au}$ départ, ils [les industriels] nous avaient dit: "oh là, là, c'est trop onéreux, vous n'y pensez pas!" Et comme il y a le voisin qui dit: "Attendez, moi, je vais être exproprié !", les industriels disent : "Ah, ben finalement, j'ai trouvé une solution, on peut aller au-delà ${ }^{20}$."»

Les négociations qui s'engagent post-MMR sont en effet essentielles: «Le PPRT, c'est une démarche itérative. Donc, nous, on fait ce qu'on peut avec la MMR, mais on n'a pas beaucoup d'arguments... La Dreal est toujours considérée comme méchante, demandant plein de choses. Pratiquement, il arrive que le contexte socio-économique demande plus que nous! Et c'est le $\operatorname{cas}^{21}$.» Si la MMR reste un «outil» pertinent ayant conduit à des réductions de risque à la source, sa portée

\footnotetext{
${ }^{17}$ Agent de la Dreal 4, mars 2015.

${ }^{18}$ Agent de la Dreal 4, mars 2015.

${ }^{19}$ Agent de la Dreal 2, janvier 2013.

${ }^{20}$ Agent de la Dreal 5, mars 2015.

${ }^{21}$ Agent de la Dreal 3, mars 2015.
} 
est néanmoins limitée par l'argument économique : «La MMR, c'est un levier, mais qui ne va que jusqu'à un certain point parce qu'il faut qu'on juge l'économiquement acceptable... Or, il y a l'économiquement acceptable et le socialement acceptable. Et l'économiquement acceptable est moins contraignant que le socialement acceptable dans certains cas $^{22}$.»

Ainsi, même si les MMR engendrent effectivement des réductions de risque à la source significatives, elles ne s'avèrent pas suffisantes en regard de la surface des territoires restant impactés: le plafond imposé par le recours au principe d'économiquement acceptable limite les efforts admis par les entreprises. Dans certains territoires, la réglementation ne permet donc pas d'obtenir une situation stabilisée. Une nouvelle phase de négociations, "post-MMR» s'ouvre alors, dans laquelle de nouvelles parties prenantes (décideurs locaux notamment), non habituellement engagées sur les questions de risques, vont faire valoir leur légitimité à participer aux décisions. Très rapidement, ces décideurs ont à leur tour recours au principe d'économiquement acceptable qu'ils font valoir pour conserver le tissu industriel non Seveso. Dès lors, l'unilatéralité et la faible discutabilité que ce principe représentait pour les entreprises Seveso sont mises en cause.

Dans de nombreux territoires en France, la stratégie de la loi fonctionne tant que les territoires affectés concernent essentiellement l'habitat. En revanche, dans les zones à forte activité industrielle, telles que celles du Havre-Gonfreville, ce n'est pas le cas et les principes de la loi Bachelot sont mis en échec. Face à la menace pesant sur la pérennité des activités économiques non Seveso (plus de 250 unités) situées dans les zones de risques appelées à être expropriées ou soumises à des mesures foncières importantes, une nouvelle phase de négociations s'ouvre, élargie à l'ensemble des décideurs locaux. Ces négociations s'appuient toujours sur le principe d'économiquement acceptable, mais qui se trouve, dès lors, pertinent tant pour les entreprises Seveso que pour les entreprises riveraines non Seveso. Ce « face à face », qui fait rapidement apparaître l'impasse dans laquelle conduisent les textes réglementaires lorsque le territoire impacté est industriel, va contribuer à assouplir discrètement la réglementation de 2003.

\section{La neutralisation de l'acceptabilité économique : vers une déformation de la réglementation}

Dans le nouveau processus de négociation qui s'engage à partir de 2010 se jouent non seulement le maintien, mais aussi le développement de l'activité

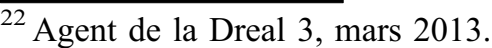

économique non Seveso. Cette démarche est d'importance dans la mesure où cette orientation, portée par le territoire (Suraud, 2013), vient heurter le principe fondateur de la loi Bachelot consistant à «faire le vide » autour des entreprises à risques. Pour les décideurs locaux, il s'agit de préserver et, aussi, de densifier le tissu économique autour des entreprises à risques en favorisant l'implantation de nouvelles activités, source d'emplois.

Une idée ambitieuse émerge progressivement, de faire de la région havraise un territoire d'accueil pour les entreprises à risques dans un contexte national où le niveau d'exigence pour l'implantation de nouvelles entreprises de ce type est de plus en plus élevé.

\section{Vers une mutualisation de la gestion des risques}

Dans la ZIP du Havre-Gonfreville se croisent différentes activités : de logistique, de chimie et pétrochimie, de fabrication et d'assemblage... ou encore de restauration et de services, dont la cohabitation est remise en cause par l'application de la nouvelle réglementation. De nombreuses unités économiques, directement liées à l'activité portuaire ou non, figurent dans la zone impactée par les risques. En délimitant des zones où est susceptible d'être interdite toute activité autre que «Seveso», le PPRT met en balance l'acceptabilité économique de la réduction des risques par les entreprises qui les génèrent et une autre sorte d'acceptabilité économique, puisque des activités voisines sont potentiellement soumises aux mesures d'interdiction prévues par le PPRT. Le gel des territoires résultant de la cartographie des risques a en outre pour conséquence de remettre en cause toute possibilité d'installation d'activités nouvelles.

La cartographie des aléas va attirer l'attention des élus sur l'étendue du problème : 70 ha à «vider», 450 ha sur lesquels doivent être financées des mesures significatives de sécurisation, que ce soit, par exemple, le renforcement du bâti ou l'étanchéisation des bâtiments en regard des risques toxiques. Pour une large part, cette superficie est occupée par des entités en «autorisation d'occupation temporaire » (AOT), attribuée par le Grand port maritime du Havre (GPMH), principal propriétaire des espaces concernés. La menace pesant sur le tissu économique non Seveso suscite un engagement des décideurs locaux pour trouver une issue à une situation qui peut sembler inextricable ${ }^{23}:$ «Le PPRT n'est pas un outil qui vise à désindustrialiser la France. C'est au contraire pour le protéger. Nous, notre rôle, c'est aussi de maintenir l'emploi et les industries. Ici, le taux de

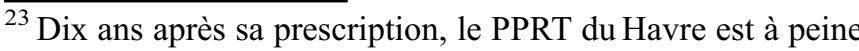
sur le point d'être adopté.
} 
chômage est à $13 \%$; c'est énorme, on ne peut pas en rajouter $^{24}$.»

Il est vrai que la ville du Havre a longtemps été tournée vers ses activités portuaires: «Le Havre, c'est d'abord un port de conteneurs ${ }^{25} \gg$ et, dans une moindre mesure, vers l'industrie de son hinterland. La création en 2000 de la Codah (Communauté d'agglomération havraise) a pour effet d'enclencher une réflexion concernant l'évolution de la ZIP, incitant les différentes communes de cette collectivité à faire converger leurs approches du port et de l'industrie. La catastrophe de l'usine AZF de Toulouse, en 2001, va catalyser ce mouvement de rapprochement entre les pouvoirs locaux et les entreprises : un service " risques majeurs » est créé au sein de la Codah, avec l'accord du préfet; puis, en 2002, une structure territoriale est formée, dédiée aux risques industriels: l'Office des risques majeurs de l'estuaire de la Seine (l'Ormes), qui vise la mise en place d'un système d'information d'envergure sur les risques.

L'Ormes, qui a un statut d'association «loi $1901 »$, reflète une propension à l'interconnexion des risques technologiques majeurs. Sa création va inciter la Chambre de commerce et d'industrie du Havre (CCIH) à se doter elle aussi d'une «commission risques industriels », regroupant essentiellement les entreprises Seveso, ce qui lui permettra d'être représentée au sein de l'Ormes. Cette association rassemble ainsi la $\mathrm{CCIH}$, la mairie du Havre, la Codah et le GPMH qui, en tant que propriétaire et gestionnaire du domaine d'implantation d'une grande partie des entreprises (notamment Seveso), peut choisir ses «locataires ».

Ainsi, bien que la gestion des risques relève, dans le principe, des services de l'État, un ensemble de décideurs locaux (mairie duHavre, Codah, CCIH et GPMH) s'empare progressivement de cette question, puisque l'issue des négociations entre Dreal et industrie, fondées sur la réglementation, n'a pas permis de limiter de façon suffisante la surface impactée. Au Havre, l'enjeu du maintien du tissu industriel non Seveso est prépondérant par rapport à celui concernant l'habitat, dans la mesure où celui-ci a fait l'objet de négociations jugées satisfaisantes par les élus. La volonté des décideurs locaux d'unir leurs réflexions sur les questions de risques dans le cadre d'une structure institutionnalisée (l'Ormes) est, en France, une situation atypique. La dynamique locale, marquée par un "accord» entre les décideurs engagés dans l'émergence de solutions permettant de maintenir les entreprises non Seveso sans mettre en difficulté économique les entreprises Seveso, conduit l'État à « déformer» la réglementation.

\footnotetext{
${ }^{24}$ Agent de la Dreal 6, septembre 2009.

${ }^{25}$ Agent du GPMH 1, janvier 2013.
}

L'Ormes va jouer un rôle fédérateur en agissant pour concilier les différentes exigences et pour initier les réflexions permettant de faire émerger des solutions bouleversant l'approche de la gestion des risques majeurs, par la désertification des territoires riverains. L'idée est de proposer une densification industrielle.

La transformation de la réglementation est rendue effective par l'adhésion volontaire de l'ensemble des décideurs locaux (CCIH, pouvoirs locaux, GPMH). L'arbitrage entre la réduction des risques à la source et le maintien - ainsi que le développement - des activités non Seveso va dépendre à la fois des rapports que les industries entretiennent entre elles (rapport de soustraitance, par exemple) et des enjeux et de la position du $\mathrm{GPMH}$, en tant que propriétaire et gestionnaire des terrains.

\section{De l'inacceptabilité économique à la déformation de la réglementation}

Deux grandes familles d'activités coexistent au Havre: portuaires (manutention, services) et industrielles, relativement comparables en termes d'emplois (respectivement: 14300 et $17300^{26}$ ). La famille des «industries» est elle-même diversifiée entre les « Seveso » (chimie et raffinage) et les autres : automobile, fabrication aéronautique, assemblage et services aux industries. La ZIP ne dépend donc qu'en partie du raffinage et de la chimie, qui représentent environ $40 \%$ de l'emploi industriel direct et $15 \%$ de l'emploi total direct $^{27}$, emploi portuaire compris.

Se pose alors la question du choix du type d'activités à privilégier. S'agit-il de maintenir et développer uniquement les activités en lien (de sous-traitance, par exemple) avec les Seveso? S'agit-il de maintenir et développer un spectre plus large d'activités industrielles non nécessairement en lien avec l'activité des entreprises Seveso?

Dans ce cadre, le GPMH va jouer un rôle essentiel. En effet, les statuts du GPMH, issus de la loi Fillon du 4 juillet 2008, portant une réforme portuaire et concernant l'adaptation aux mutations du transport maritime européen et mondial ${ }^{28}$, lui fournissent des leviers pour pérenniser, ou non, les entreprises implantées sur la ZIP,

${ }^{26}$ Insee, Clap (validité 2010), GPMH-AURH, n 132, février 2013.

${ }^{27}$ Ibidem.

${ }^{28}$ La loi Fillon comporte quatre grands axes dont «l'évolution des missions des sept ports autonomes rebaptisés "grands ports maritimes"». Cet axe recentre les GPM sur les fonctions d'aménageur du domaine portuaire et s'inscrit dans une perspective conciliant développement économique, respect de l'environnement et soutien au transport multimodal. 
sur les terrains dont il est propriétaire ${ }^{29}$. La loi Fillon a organisé la réforme des «ports autonomes» et leur transformation en grands ports maritimes. Elle a eu pour effet de faire sortir le port de sa fonction administrative (la gestion des terrains qui lui sont confiés par l'État), pour lui accorder plus d'autonomie, donc plus de marges de manœuvre dans la gestion de son domaine, devenu sa propriété. Ainsi, la constitution du GPMH le conduit à passer d'une fonction de gestionnaire de terrains à celle de gestionnaire d'une zone industrialo-portuaire ${ }^{30}$. Sous l'impulsion du GPMH, la carte des risques va être redessinée.

Comme tout propriétaire, le GPMH est amené à étudier les conditions de rentabilisation de son espace, donc la compatibilité des activités à risques et des autres activités, incluant celles qui sont amenées à être développées. Par exemple, le «projet éolien », représentant plusieurs centaines d'emplois potentiels dans la fabrication de matériels et la production d'énergie, apparaît porteur d'avenir, mais il nécessite des terrains disponibles dans la ZIP.

Bien que le GPMH n'ait pas accepté de mener une action institutionnalisée - souhaitée par la Dreal - dans la prévention des risques, il va intervenir fortement dans la gestion des risques en exigeant un traitement spécifique des zones industrielles non Seveso, dont les activités du port. L'implication du GPMH dans la gestion du risque ne peut cependant qu'être indirecte, puisque, hormis la prise en charge de la sécurité des activités portuaires jusqu'au bord de quai (exceptionnellement jusqu'aux bateaux), le GPMH n'a pas la compétence «risques technologiques majeurs ». Il ne peut s'introduire dans une usine pour l'inspecter, pas plus qu'il ne peut formellement s'immiscer dans la procédure propre aux études de dangers, qui reste une prérogative de l'administration des installations classées. L'intervention du GPMH n'en sera pas moins effective.

L'enjeu est double. D'une part, obtenir de la part des entreprises Seveso des réductions de risques à la source, bien au-delà de ce qui a été obtenu par l'application de la réglementation, pour libérer de l'espace et le rentabiliser. En effet, la réglementation s'appuyant essentiellement sur la MMR, lorsque celle-ci est établie et lorsque les entreprises sont conformes à cette norme, il se peut que les territoires impactés soient encore jugés trop étendus. Néanmoins, la réduction «supplémentaire» de risques à la source ne peut passer que par des négociations au cas par cas, sur des bases qui échappent aux calculs propres à

\footnotetext{
${ }^{29}$ Cependant, des établissements ont en propre leur foncier comme, par exemple, Renault à Sandouville, jouxtant un établissement Seveso AS ou encore Aircelle à Gonfreville, jouxtant deux établissements Seveso AS.

${ }^{30}$ Entretien avec un responsable du GPMH 2, février 2015.
}

la construction des MMR. D'autre part, il s'agit d'obtenir, de la part de l'État, un assouplissement de la réglementation de l'aménagement des zones de risques.

Pour autant, le GPMH et les décideurs locaux admettent que les réductions «supplémentaires» des risques à la source pour diminuer la surface des territoires impactés doivent être limitées dès lors que les entreprises Seveso indiquent jouer leur survie. Les décideurs locaux vont donc, simultanément aux négociations locales, s'engager auprès de la DGPR dans une demande de transformation de la réglementation de 2003 en vue de flexibiliser les règles d'aménagement des zones de risques.

Cette démarche conduit vers un nouveau complément de réglementation prenant la forme de «notes » publiées en 2011 et 2012, dont la valeur réglementaire, notamment en termes de responsabilité, laisse durablement perplexes des décideurs et experts locaux et, même, des agents de l'administration ${ }^{31}$. Néanmoins, dans la mesure où ces notes permettent d'aménager la réglementation, aucune discussion fondamentale ne s'engage sur leur portée juridique et leur fragilité éventuelle en cas de recours juridique.

La note de mai 2011 est relative au «traitement des activités économiques» et stipule que ${ }^{32}$ : «Certaines activités présentent des caractéristiques telles que leur délocalisation peut soit engendrer des conséquences sur le fonctionnement technique ou économique de la zone, voire remettre en question la viabilité des entreprises à l'origine du risque présentes... Ces activités peuvent être classées dans l'un des cas suivants : activité présentant un lien direct avec l'établissement à l'origine du risque, activité prestataire pour l'établissement à l'origine du risque ou activité participant au service portuaire. » $\mathrm{La}$ note de janvier 2012 est relative à l'«application de la doctrine PPRT dans les zones portuaires ${ }^{33} \gg$ et stipule que «[...] sur le domaine public portuaire, certaines conditions doivent être respectées... [conduisant à ce que] dans certains cas, les mesures foncières ne peuvent pas être envisagées et d'autres solutions doivent alors être mises en œuvre». Ces deux notes amorcent une inflexion significative des modalités de mise en œuvre de la loi Bachelot. Elles visent à introduire de nouveaux critères et éléments de négociation sur le maintien des activités riveraines : «Les activités économiques sont par nature très variées et présentent des caractéristiques propres qu'il convient de prendre en compte... Ces caractéristiques entraînent qu'elles ne peuvent être

\footnotetext{
${ }^{31}$ Entretiens multiples.

${ }^{32} \mathrm{http}: / / \mathrm{www} . n o r m a n d i e . d e v e l o p p e m e n t-d u r a b l e . g o u v . f r /$ IMG/pdf/note traitement des activites economiques.pdf.

${ }^{33} \mathrm{http}$ ://www.normandie.developpement-durable.gouv.fr/ IMG/pdf/note_activites_ports-01-12.pdf.
} 
traitées de façon globale et qu'il est indispensable de différencier leur traitement ${ }^{34}$. » Par les notes de 2011 et de 2012 est ainsi admise la possibilité de déroger aux principes fondateurs de la loi Bachelot en autorisant une implantation d'activités dans des zones qui auraient dû, $a$ priori, être "vidées», possibilité formulée explicitement: "l'application des mesures foncières sur ces activités ne paraît pas pertinente » quand des activités sont connectées aux entreprises Seveso ou quand elles sont portuaires ou encore quand elles relèvent «d'activités avec protection possible $\mathrm{e}^{35} »$.

Autrement dit, alors que la loi Bachelot avait pour enjeu de faire le vide autour des établissements à risques, les différentes inflexions qui lui ont été données ont conduit, dans des zones fortement industrialisées telle que la zone industrialo-portuaire du Havre, à reconsidérer cette ambition à la baisse et à prendre le parti de densifier les territoires riverains des «Seveso». Ce renversement de principe (passer d'un vide autour des Seveso à une densification de l'activité industrielle riveraine) marque un retournement significatif des principes de la loi. De ce point de vue, l'État s'arrange avec lui-même, à travers des textes dont la valeur réglementaire reste à établir, pour déformer sa propre réglementation.

\section{Conclusion}

La loi Bachelot s'est inscrite dans la perspective générale de réduire la vulnérabilité environnementale des populations riveraines. En pratique, elle est amenée à devoir traiter, sans leviers réglementaires établis, de questions relatives à la vulnérabilité socio-économique d'un espace industriel. Ainsi, la réglementation formule des exigences en termes de niveau de risques vis-à-vis des entreprises, tout en leur laissant, en même temps, la capacité de limiter ces exigences au titre de leur « inacceptabilité économique».

Il en résulte qu'une réglementation des risques peut non seulement fabriquer des risques (Gilbert, 2003), mais aussi une concurrence entre intérêts économiques. La loi Bachelot se voit débordée par elle-même en raison de ses implications imprévues sur la coexistence, voulue, des activités Seveso et non Seveso existantes et à venir.

Il reste à évaluer la façon dont cette confrontation entre enjeux économiques pèsera, à terme, sur les principes de gestion des risques en France. Si, après la catastrophe d'AZF, ils se sont révélés ambitieux, en pratique, dans certains territoires très fortement industrialisés, ils se sont, au moins partiellement, dissous dans l'économique.

\footnotetext{
${ }^{34}$ Note de mai 2011, «Traitement des activités économiques ». ${ }^{35}$ Ibidem.
}

\section{Références}

Bonnaud L., 2005. Au nom de la loi et de la technique, Politix, 69, 131-161, https://doi.org/10.3917/pox.069.0131.

Bonnaud L., Martinais E., 2005. Des usines à la campagne aux villes industrielles : la cohabitation ville/industrie saisie à travers l'histoire du droit des établissements classés, https://journals.openedition.org/developpementdurable/ 749 ? \&id $=749$.

Centemeri L., 2010. The Seveso disaster legacy, in Armiero M., Hall M. (Eds), Nature and History in Modern Italy, Athens $(\mathrm{OH})$ : Ohio University Press \& Swallow Press, 251-273.

Chabbal J., 2005. Le risque invisible. La non-émergence d'un problème public, Politix, 70, 169-195, https://www.cairn. info/revue-politix-2005-2-page-169.htm.

Frère S., Gibout C., 2012. De la formation à l'incertitude à la gouvernance par l'incertitude. Les acteurs locaux face au Plan de Prévention des Risques Technologiques (PPRT) de la zone industrialo-portuaire de Dunkerque, Rapport final du programme CDE (Concertation, Décision, Environnement), Ministère de l'Écologie, http://www.concertationenvironnement.fr/documents/RF/FrereGibout_Synthese. pdf.

Fressoz J.-B., 2012. L'apocalypse joyeuse. Une histoire du risque technologique, Paris, Le Seuil.

Gilbert C., 2003. La fabrique des risques, Cahiers internationaux de sociologie, 114, 1, 55-72, https://www.cairn. info/revue-cahiers-internationaux-de-sociologie-2003-1page-55.htm.

Gralepois M., 2011. L'improbable préemption des territoires à risque industriel majeur, Géocarrefour, 86, 3-4, 271-280, http://journals.openedition.org/geocarrefour/8492.

Lascoumes P., 2011. Les normes juridiques et les normes techniques de l'inspection des installations classées pour la protection de l'environnement, Annales des Mines-Responsabilité et environnement, 62, 2, 30-34, https://doi.org/ 10.3917/re.062.0030.

Le Roux T., Letté M., 2013. Débordements industriels. Environnement, territoire et conflits. XVIII ${ }^{e}-X X I^{e}$ siècle, Rennes, Presses universitaires de Rennes.

Martinais E., 2007. L'administration des risques industriels: entre renouvellement et stabilité, Regard sur l'actualité, 328, 25-37, https://hal.archives-ouvertes.fr/hal-00507147.

Martinais E., Galland J.-P., 2010. La prévention des risques industriels en France. Bilan et perspectives, Actes de séminaire Les enjeux d'une gestion territorialisée des risques technologiques. Spécificités françaises et mise en perspective internationale, PUCA, LATTS, EVS-RIVES, 2007-2009, Paris, https://halshs.archives-ouvertes.fr/ halshs-00619234/.

Propeck-Zimmermann E., Saint-Gérand T., Bonnet E., 2007. Probabilités, risques et gestion territoriale : champs d'action des PPRT, Géocarrefour, 82, 1-2, 65-76, http://journals. openedition.org/geocarrefour/1473.

Rasse G., 2009. Les plans de prévention des risques technologiques au prisme de la vulnérabilité. Le point de vue du juriste, Thèse de doctorat, Paris, École nationale 
supérieure des Mines, https://pastel.archives-ouvertes.fr/ tel-00363570.

Suraud M.-G., 2013. La thématisation des risques industriels majeurs en France : la concurrence «participative» comme enjeu, VertigO, 13, 3, http://journals.openedition.org/ver tigo/14390.

Treich N., 2008. L'analyse coût-bénéfices en dix questions, Les Cahiers de la sécurité industrielle, 3.

Citation de l'article : Suraud M.-G., 2019. La mise en œuvre d'un plan de prévention des risques technologiques: acceptabilité économique vs réduction des risques. Nat. Sci. Soc. 27, 3, 267-277. 